\title{
Enjeux de la médiation comme révélateur de l'interprétation des enfants, en contexte muséologique
}

Mediation as revelation of how children interpret art in museums: an issue

\section{Thérèse Martin}

\section{(2) OpenEdition}

\section{Journals}

Édition électronique

URL : http://journals.openedition.org/communicationorganisation/5121

DOI : 10.4000/communicationorganisation. 5121

ISSN : 1775-3546

Éditeur

Presses universitaires de Bordeaux

Édition imprimée

Date de publication : 1 décembre 2015

Pagination : 167-184

ISSN : 1168-5549

Référence électronique

Thérèse Martin, «Enjeux de la médiation comme révélateur de l'interprétation des enfants, en contexte muséologique », Communication et organisation [En ligne], 48 | 2015, mis en ligne le 01 décembre 2018, consulté le 04 janvier 2020. URL : http://journals.openedition.org/

communicationorganisation/5121 ; DOI : 10.4000/communicationorganisation.5121 


\title{
Enjeux de la médiation comme révélateur de l'interprétation des enfants, en contexte muséologique
}

\author{
Thérèse Mortin
}

\section{Introduction}

Les Sciences de l'Information et de la Communication sont propices pour étudier les pratiques culturelles sous des angles diversifiés en fonction des objets questionnés par les chercheurs, pouvant avoir trait entre autres à la culture médiatique (TV, presse, internet...), aux lieux d'expositions, ou aux industries culturelles. Comme en témoignent déjà en 1998, les articles du numéro 21 intitulé «Médiations culturelles : dispositifs et pratiques» de la revue Études de Communication ${ }^{2}$, mais aussi d'autres revues ou colloques qui ont suivi - notamment les actes du colloque «Dispositifs et médiation », le Dossier 2010 «La (les) médiation (s) en SIC " ${ }^{3}$, ce sont sur des mondes ou des « lieux de médiation » hétérogènes que peut porter l'analyse des pratiques concrètes de médiation culturelle. Les approches choisies pour étudier ces pratiques sont tout aussi variées (sémiologie, analyse de discours, anthropologie ou socio-économique). Parmi les problématiques soulevées autour de la notion de médiation, celle se rapportant aux dispositifs de médiation mettant en exergue les interactions suscitées, et celle concernant la construction de sens

1 Thérèse Martin est chercheure en Sciences de l'Information et de la Communication, et membre associée du laboratoire Médiation Information Communication Art (MICA) de l'Université Bordeaux 3. Après s'être intéressée à l'usage du multimédia interactif et ses incidences sur les processus cognitifs des enfants, elle s'est orientée vers un autre média, l'exposition, pour étudier son appropriation par les enfants dans le cadre des loisirs. Ses travaux de recherche sont centrés sur les logiques d'interprétation des enfants, in situ au cours de leur expérience de visite, dans l'espace de médiation des musées de sciences et d'histoire ; therese.martin@wanadoo.fr

2 Entre autres : un magazine féminin (Giet), des émissions documentaires de télévision (Gellereau), la promotion des films (Croquet), l'accueil du public en entreprise (De la Broise) dans la revue Études de communication.

3 La revue MEI, n 19, "Médiation et médiateurs », le monde des arts de la scène avec la danse et le théâtre contemporain (Bordeaux), la presse sur internet (Lancien).

Le colloque "les dispositifs de médiation: théories, dispositifs et enjeux », 19-20 novembre 2009 à Metz-Nancy. coord. par AJC CREM

Les sept articles (coordonnés par Jean Caune) du dossier 2010 de la revue Enjeux de l'Information et de la Communication recouvrent des pratiques diverses (documentaire, politique, médiatique, psychiatrique...). Les questions visent toutes à interroger la pertinence de la notion de médiation pour comprendre les pratiques de communication, les changements sociotechniques et le rôle des acteurs, qualifiés de médiateurs. 
par les acteurs dans des pratiques de communication (le phénomène de visite d'exposition compte parmi ces pratiques) ont pu attirer notre attention. Et pour ce qui est du contexte muséologique, l'interrogation porte sur les manières dont s'instaure une relation entre le monde du visiteur et le monde scientifique par l'intermédiaire de l'exposition, considérée comme dispositif médiatique (socio-symbolique) et comme espace de production langagière, si lon se réfere à Jean Davallon (Davallon 2005).

À partir de notre recherche ${ }^{4}$ située en contexte de médiation culturelle, et de son terrain particulier les institutions muséales de sciences, les visites d'exposition de visiteurs-enfants (Martin 2011), nous envisageons de montrer en quoi la notion de médiation tient une place importante, tant sur le plan de la méthode que sur le plan de la théorie, pour approcher les façons dont les visiteurs-enfants s'approprient l'exposition. Ce qui est abordé ici, c'est l'articulation entre la notion de médiation convoquée dans la conception de notre dispositif méthodologique et celle de médiation comme concept précieux pour mettre au jour les démarches interprétatives des enfants lors de leur visite d'exposition, sur le temps de loisirs. Plus précisément, nous proposons de montrer comment la notion de médiation dans un contexte de muséologie des sciences contribue, de façon primordiale, à la fois à mettre en lumière les processus d'interprétation des enfants en train de se dérouler grâce à une méthode consistant en un dispositif communicationnel ("la mise en situation de rôle de guide»), et à analyser les données issues de la production de récits de médiation, à les conceptualiser afin de dégager des figures d'enfantsinterprètes selon les trois formes de la médiation (comme transition, comme lien et comme intermédiaire).

Afin de mieux saisir les enjeux de la médiation et leur ancrage dans notre terrain d'expérimentation, nous traçons un aperçu du fil conducteur de notre démarche. Nous commencerons par resituer le contexte, avec la définition du musée comme média, celle de l'exposition en tant que dispositif médiatique mis en œuvre par les concepteurs. Puis nous orientons notre regard sur l'espace de médiation - l'entre-deux - entre exposition et visiteurs, dont la particularité est de pouvoir être considéré comme un environnement où sont offerts des « dispositifs » (Berten) suscitant des réactions, de l'interaction de la part des visiteurs. Si bien que cet espace de médiation est propice à la production d'expériences, à commencer par l'expérience esthétique selon la perspective phénoménologique. Nous verrons qu'en contexte de médiation culturelle, il est un lieu de construction de sens. Les démarches interprétatives des enfants seront approfondies à partir de ce qu'ils "fabriquent ", ce qui inscrit ainsi plus largement notre recherche dans le champ de la culture " ordinaire »

4 Notre recherche doctorale empirique s'est attachée à comprendre de manière exploratoire les démarches d'interprétation des enfants inscrites dans leur expérience de visite dans les musées de sciences, dans le cadre des loisirs. Elle a conduit à mettre en lumière les logiques d'interprétation des enfants et à dégager des modes d'appropriation de l'exposition. 
(M. de Certeau). La médiation enlien avec le dispositif est rendue visible à travers notre dispositif méthodologique innovant, "la mise en situation de rôle de guide " (proposée en fin de visite en famille) conduit à la production de récits de médiation puisque les enfants jouent le rôle de "médiateur ". Quant au plan théorique, la notion de médiation intervient dans l'analyse de ces récits en référence aux trois formes de la médiation, la médiation comme transition, comme intermédiaire et comme lien (Gellereau 2004). Ce qui a permis de dégager des modes d'appropriation de l'exposition par les enfants.

Au cours de cet article, nous prendrons soin de fournir le cas échéant, des éléments relatifs à notre terrain d'expérimentations et aux récits des enfants dans le but de mieux illustrer les enjeux de la médiation. Pour découvrir l'ensemble de la démarche de notre recherche, une synthèse accompagnée des paroles d'enfants en illustrations est présentée dans la revue "Communication » de l'Université Laval au Québec (Martin 2012).

\section{La visite, une rencontre entre le visiteur et le média-exposition}

Nous nous interrogeons dans un premier temps sur les caractéristiques de ce média-exposition afin de mieux appréhender comment il est interprété par ses usagers. C'est E. Véron $(1983)^{5}$ qui a initialement tenté de conceptualiser l'exposition comme média, puis J. Davallon (article réactualisé publié en 1992) a renouvelé sa définition en précisant ses caractéristiques. Selon ce chercheur en muséologie, le point de départ de l'approche du musée comme média est la relation sociale qui s'établit au cours de la visite entre des acteurs sociaux et un ensemble d'objets de musée (ou musealia) 6 étant reconnus comme dignes d'être conservés et présentés. Ainsi penser le musée comme média revient à considérer le musée comme espace de rencontre entre des musealia et des acteurs qui sont invités à regarder, contempler, découvrir, s'instruire, entrer en contact avec ces objets. L'exposition (la technologie du musée) quant à elle, organise l'espace d'une rencontre et règle la relation qui s'établit entre les objets et le public. Notons que l'exposition est spontanément liée au musée ${ }^{7}$. Par mesure de simplification, nous attribuerons la notion de « média » à l'exposition. En définitive, le média est considéré comme la mise en place d'un dispositif communicationnel, à la fois technique et social qui instaure un monde de langage dans lequel un récepteur et des objets vont se trouver mis

\footnotetext{
5 Regrettant qu'on se place généralement dans une optique de production « quand on parle de médias » et que le dispositif technologique soit indépendamment envisagé des phénomènes de circulation et de réception des messages, É. Véron considère que les conditions d'accès, de reconnaissance des messages sont à prendre en compte. En 1982, il réalise la première étude avec le suivi des visiteurs pour essayer d'observer au plus près leurs pratiques, dans une exposition photographique à la BPI du Centre Pompidou. Voir aussi É. Véron (1992). 6 «Objets de musée » (des musealia) : pris au sens très ouvert, couvrant des choses issues de la nature ou de l'industrie des hommes, ayant forme d'objets, d'images ou de discours. Il peut s'agir aussi bien d'objets conservés par le musée que d'outils de présentation (Davallon 2005 : 235).

$7 \mathrm{Il}$ est bien entendu que c'est au musée que les règles culturelles, qui normalisent la mise en forme des collections et leur agencement visible, sont institutionnalisées, et que la « technologie des musées est constituée par l'exposition» selon J. Davallon (1992: 230).
} 
en relation. Il permet aux visiteurs d'entrer en relation avec le monde auquel appartiennent ces « objets ». Ce dispositif rend ainsi possible les opérations d'interaction, de discours, de lien social. Cette perspective proposée par J. Davallon (2003 : 27) a contribué de façon notoire à la progression des études liées au phénomène de visite. C'est à partir de cette volonté de favoriser cette relation, que E. Caillet (1995) quant à elle, a insisté sur l'accompagnement des publics qui a pris de l'importance avec le développement de la notion de " passage » pour transmettre la culture, en vue d'une démocratisation culturelle. Dans les musées de sciences, à propos des types de communication à l'œuvre, le chercheur R. Silverstone (1998) considère quant à lui le musée en termes d'espaces dans lequel des objets et leur mise en scène sont offerts au public, proposant ainsi un " éventail de possibilités discursives ». Les espaces discursifs proposés par ce chercheur sont l'espace de la rhétorique, l'espace $\mathrm{du}$ jeu et celui de la performance "dans lesquels les visiteurs participent à la construction du sens ».

\section{Le contexte muséal comme lieu de médiation culturelle et de construction de sens}

Dans un deuxième temps, nous portons intérêt à cet espace de rencontre à travers la prise de contact, les interactions des visiteurs avec ce dispositif médiatique et les manières dont ils construisent du sens à des fins d'appropriation de l'exposition.

Ces questions d'appropriation sont évoquées par B. Lamizet et A. Silem, quand ils définissent la "médiation " dans le dictionnaire encyclopédique des Sciences de l'Information et de la Communication. L'appropriation est rendue possible pour ceux qui font partie de l'espace social, grâce à la communication qui exerce une fonction de médiation dans l'espace social, par l'intermédiaire de structures, de formes du langage et de différents codes. Plus précisément la médiation est définie comme une "notion perméable », qui amène à s'interroger "sur les questions de structuration et d'appropriation, qu'il s'agisse de la production ou de la diffusion de la culture » si l'on se réfère aux travaux de M. Gellereau (2004 : 43).

La pratique de visite s'inscrit plus largement dans un cadre de médiation culturelle.Celle-ci est entendue selon le premier aspect dela définition proposée par B. Dufrêne et M. Gellereau, à savoir la médiation comme «situation de médiation ". Cet aspect prévoit d'analyser la manifestation elle-même, soit le niveau le plus apparent de la médiation, et peut concerner notamment l'exposition (Dufrêne, Gellereau 2004 : 199). La question importante soulevée à propos de la médiation culturelle est celle de la construction de sens, selon M. Gellereau. Comment les publics interprètent-ils les « objets » présentés ? Des éléments de réponse sont apportés dans son article "Pratiques culturelles et médiation ". La notion de médiation, vue sous l'angle de la construction du sens (le processus interprétatif) «se fonde sur le fait que le sens n'est pas 
immanent aux objets mais se construit par des sujets interprétants dans des processus, grâce à des langages et des dispositifs » (Gellereau 2007 : 29). Par ailleurs, c'est au cœur de certaines approches de la médiation que se situe la notion d'interprétation, "puisque [...] l'intérêt se porte sur la part active des visiteurs ou des publics dans la construction du sens des œuvres ». (Gellereau $2007: 32$ ).

Si l'on se situe en contexte muséal, le dispositif médiatique de l'exposition s'appuie sur des stratégies de communication visant à mettre en scène des objets concrets. Le lien entre producteur et visiteur est alors établi par J. Davallon (2005 : 21) de la manière suivante : « ces visiteurs sont mis en relation avec un univers symbolique (le "monde utopique") [...] ». Ce « monde utopique ", imaginé, donne lieu chez le visiteur à des activités d'interprétation. En effet, « le monde utopique que nous construisons à partir des objets [...] peut [être] considér[é] comme un monde imaginé, puisque intervient, de la part du visiteur, une activité d'interprétation qui donne à cette construction un caractère subjectif ou peu contrôlé ». (Davallon $2005: 30$ ).

Dans le cadre des pratiques culturelles muséales des enfants, c'est donc sur cette « situation de médiation " propre au contexte de médiation culturelle que nous focalisons notre attention pour étudier ce qui se joue dans cet entre-deux (ou espace de la médiation), c'est-à-dire entre le dispositif médiatique créé par les concepteurs (ou l'espace «synthétique » ainsi nommé par J. Davallon, 2005 : 170), doté de qualités communicationnelles, et les visiteurs. Nous nous attachons à la part active de ces visiteurs-enfants dans leurs processus de construction du sens. Il s'agit de pouvoir cerner leurs démarches interprétatives qui participent à la construction de leur « monde utopique », ceci en lien avec chacun des espaces discursifs de l'exposition (proposés par R. Silverstone, 1998).

\section{Médiation, dispositif et vécu d'expériences}

Nous envisageons à présent de mieux cerner comment se caractérise cet espace de médiation exploré par les visiteurs. Les chercheurs A. Klein et J.-L. Brackelaire (1999 : 68) considèrent que les dispositifs poseraient le problème de la "médiation culturellement nécessaire pour que les gens puissent être en prise sur leur vie dans toutes ses dimensions " ou autrement dit pour qu'ils puissent se relier aux mondes avec lesquels ils sont en contact. Selon ces auteurs, le recours à la notion de dispositif participerait de cette nécessité où nous sommes tous de " créer et d'avoir à notre disposition des espaces de (re)création et d'appropriation de l'expérience » en nous inscrivant dans l'échange. Ce seraient des lieux de reprise, de production de l'expérience, et donc d'implication dans la vie sociale que constitueraient ces dispositifs. Si cette création d'espaces d'appropriation de l'expérience est possible par l'intermédiaire du dispositif, A. Berten quant à lui apporte des précisions sur les manières dont les individus disposent de ces espaces (ou plutôt de 
leur environnement) au point de savoir en saisir les potentialités. Pour ce philosophe belge, penser le dispositif, c'est « penser la manière la plus naturelle dont l'individu est parfaitement à même de se situer dans son environnement, de l'apprivoiser, de le modifier, de l'ingérer et de le régurgiter » (Berten 1999: 43). Les " dispositifs " désignent chez cet auteur les objets transitionnels qui s'y disposent et s'y rendent disponibles. Dans le cas de l'exposition, où se mêlent technique (mise en scène du monde représenté par les concepteurs) et symbolique (le monde que se représentent les visiteurs ou " monde utopique " selon J. Davallon), ce concept d'environnement a tout à fait sa place. Il peut être défini à la fois par les ressources qu'il met à disposition, par leur agencement et par les potentialités des actions qui peuvent être réalisées par leur intermédiaire (apprivoiser, modifier, contempler, s'approprier...). Alors réfléchir sur la médiation reviendrait à observer plus attentivement les manières dont les individus prennent leur place dans cet environnement cognitif (au sens large du terme) comme espace potentiel de développement des compétences, des savoirs, des savoir-faire (Berten 1999). Ce qui se rapproche de la vision des auteurs A. Hennion, S. Maisonneuve, É. Gomart pour qui « le véritable pouvoir des dispositifs tient à leurs capacités à faire émerger une prolifération de compétences ", mettant ainsi l'accent sur le caractère productif du « dispositif » $(2000: 168)$.

Pour revenir à notre expérimentation, les visiteurs-enfants sont en mesure de reprendre partiellement leur première expérience de la visite en famille, ou d'autres expériences antérieures, ou bien encore de faire appel à d'autres médias. En devenant le «guide " pour le chercheur, ils (re)-créent une expérience dans la mesure où ils sont acteurs (ils prennent en main la situation de médiateur de l'exposition) et ajustent leurs premières représentations de l'exposition par rapport à la visite en famille, en vue de rendre explicite leur récit de médiation. Cela rend possible la mise en évidence des opérations entrant en jeu dans leur démarche interprétative. Cet environnement de diffusion de savoirs (mission de l'exposition de sciences) constitue bien un cadre potentiel pour vivre des expériences.

Les caractéristiques heuristiques de la notion de « dispositif » ont contribué à conforter le choix de la création d'un dispositif méthodologique qui puisse montrer la diversité et la richesse des opérations mises en œuvre par les visiteurs en lien avec le monde «synthétique » construit par les concepteurs et qui ne se limite pas à une appropriation de connaissances. Les différents liens effectués par les visiteurs-enfants quand ils interprètent ont pu mettre en exergue que " la médiation est culturellement nécessaire » : le lien entre l'exposition et leur propre culture par l'intermédiaire d'autres médias, l'école (la référence à la maîtresse, à ce qui a été appris à l'école), les connaissances des copains.

Un visiteur-enfant fait allusion à sa petite enfance, période où il situe les souvenirs du début de sa passion pour les dinosaures : 
$\mathrm{Vi}$ : oui, quand j'étais petit j'en étais amateur (...) déjà tout petit, à quatre ans, je posais des questions aux parents

Un autre visiteur-enfant est en mesure de reconnaître le Tricératops grâce à un copain :

Lu : oui , c'est un copain, il me l'avait expliqué, parce qu'il a trois cornes.

Ou se souvient des propos de la maîtresse d'école à propos de caractéristiques du TRex :

Lu : ah la maîtresse nous l'a dit

Un autre encore, un autre se souvient d'un film sur les dinosaures :

Vi : d'après les films je pense qu'il marche comme ça... en sautillant

Les visiteurs-enfants ont fortement envie de découvrir, de connaître, comme le montrent les principales opérations à l'œuvre quand ils découvrent l'exposition, à savoir l'observation, le questionnement et la manipulation dans le cas des activités interactives.

Mais aussi ils peuvent être passionnés par la scénographie de l'exposition, en particulier par les effets sonores ou par une fausse ombre :

Sa : [...] c'est quand t'as des bruits et tu sais pas d'où ils viennent, [...] t'entends des grincements de porte, euh et après, après on va dire des fantômes et tout ça

Sa : et là c'est, on croit que quelqu'un dort, mais en fait c'est une boule qui fait provoquer le ronflement

Sa : que c'est amusant et euh...c'est amusant, en même temps il y a des énigmes comme celle-ci l'ombre il faut savoir de qui elle est et de quoi et plusieurs choses. [... ]. On fait voir que c'est de la grenouille, mais ... ça l'est pas. ... Enfin j’ai aimé.

D'autres s'interrogent tout en s'appuyant sur leurs connaissances antérieures :

Fa : s'ils l'avaient trouvé mort, il aurait peut-être pas eu de blessures. ... Et puis je sais que dans certains documents que j'ai vus, beaucoup de dinosaures qui tuaient d'autres dinosaures ils les gardaient pour eux, ils le laissaient pas pour les autres.

\section{Appréhender l'expérience de visite à partir de l'expérience esthétique}

Nous avons emprunté la perspective phénoménologique pour mieux comprendre la relation établie entre les visiteurs et le " monde ", celui de l'exposition ("monde synthétique »). C'est " par le corps que le monde est perçu, qu'il vit, agit, ressent ", et l'expérience est une affaire d'« interactivité de l'organisme et de l'environnement » (Dewey 2006 : 13). Pour J. Dewey, l'expérience esthétique a été un point de départ pour étudier l'expérience : « La construction du monde social ne s'établit pas seulement dans son intelligibilité, mais aussi par un ajustement sensible ». A partir de là, nous estimons que pour 
comprendre l'expérience vécue par les visiteurs-enfants, il convient de porter intérêt à leur expérience esthétique. Pour répondre à l'invitation de J. Caune $(2008)^{8}$, il s'avère pertinent de prendre en compte la connaissance que procure l'expérience esthétique, marquée par de fortes charges émotives, dont les souvenirs peuvent être imprégnés, et de réfléchir sur la médiation esthétique.

En contexte muséologique, la médiation sensible est particulièrement développée dans la conception des expositions temporaires à thème. Comme le souligne le muséologue A. Delarge (2001 : 58), la scénographie des expositions temporaires, mettant en exergue les stratégies communicationnelles, présente certaines caractéristiques dont celle de créer un environnement « sensible » qui propose une ambiance, voire qui génère des émotions ou des sensations. Selon lui, l'interprète (ici le concepteur) se place dans une démarche de "médiation entre des visiteurs et des objets : il cherche à établir un lien direct entre le monde de l'objet et celui du visiteur ", et tente d'en révéler les significations profondes et les relations avec leur environnement. Ce type d'exposition s'inspire des objectifs assignés à l'interprétation qui ont été mentionnés déjà par le journaliste F. Tilden (1957), auteur de l'ouvrage sur l'interprétation à destination des animateurs, dans le cadre de la mise en place des Centres d'interprétation au Canada. D'après lui, ces objectifs reviennent à savoir donner l'envie de découvrir, d'expérimenter, de comprendre, de s'approprier de nouvelles connaissances, de questionner.

Pour ce qui concerne nos expérimentations, elles ont eu lieu dans deux expositions ayant comme visée commune d'intéresser les visiteurs-enfants aux sciences par une médiation sensible. Il s'agit de l'exposition "Le festin des dinosaures » (au Palais de la découverte) dont la médiation sensible vise à toucher par l'émotion; de l'exposition "Ombres et lumière » de la Cité des enfants à la Cité des Sciences et de l'Industrie, qui ambitionne de sensibiliser le jeune public à la dimension artistique par l'association des sciences et des arts. Ces paroles d'enfants témoignent de leur sensibilité à cette médiation sensible :

\section{Dans l'exposition «le festin des dinosaures"}

$\mathrm{Ni}$ : c'est impressionnant comment ils ont fait tous les montages avec les Tyrannosaures Rex. Ils bougent tous, les dinosaures

\section{Dans l'exposition « Ombres et lumière »}

$\mathrm{Li}$ : Je ne sais pas comment il a fait, mais c'est très impressionnant.

Ju :j’aime surtout quand il y a plein d'ombres.

8 « Les formes artistiques ne sont plus seules à témoigner de la vie psychique, à mettre en jeu l'imaginaire, à mobiliser les affects, à produire de la jouissance esthétique. Il convient également de réfléchir sur les médiations qui empruntent à l'expérience artistique sa capacité d'influencer notre perception, de conditionner notre imaginaire, de mobiliser nos émotions et notre implication affective ». (Caune $2008: 65$ ). 
Ce sont par exemple des souvenirs d'expériences antérieures que les enfants se remémorent, tel un film sur les dinosaures vu à la maison, simulant la disparition des dinosaures, et ressentie comme dramatique.

$\mathrm{Ch}$ : je l'ai vu c'est papa qui l'a, alors moi je l'ai regardé, c'était joli... J'ai pas très bien aimé la fin ... parce que il y avait que les os...

Une nouvelle expérience de visite avec le chercheur est l'occasion de revivre des sentiments de plaisir ressentis lors de la découverte d'une activité et qui sont aussi partagés avec leur visiteur. Nous citons notamment l'activité de l'« ombromaton » (exposition "Ombres et lumière ») qui propose de réaliser l'ombre de son profil et d'emporter une version papier à retrouver sur internet. Leur sensibilité esthétique et leur besoin de partager sont donc mis à profit pour interpréter.

Le plaisir d'écouter un poème qui l'a touché :

Ju : c'est joli le poème du petit monsieur [...] [il parle] des nuages, du soleil, un moment il dit «ami».

\section{Médiation, culture « ordinaire » et interprétation}

Nous voulons montrer à présent, comment notre recherche s'inscrit plus largement dans le cadre de la culture « ordinaire ». Si J. Davallon en 1998 prônait (déjà) l'ouverture des musées en tant qu' " outils de culture ${ }^{9}$, force est de constater que les actions de ces institutions restent encore de nos jours majoritairement dirigées vers une action éducative auprès du public scolaire, dans un souci d'apprentissage (Davallon 1998 : 399), comme en témoignent les nombreuses études en muséologie réalisées dans le cadre scolaire. Dans une perspective similaire, les chercheurs J. Eidelman, M. Van Praët revendiquent « la spécificité d'une culture muséale » pour les musées de sciences, reconnaissant que les services éducatifs des musées reproduisent à tort l'École (Eidelman,Van Praët $2000: 7)^{10}$.

Face à l'impact de la pédagogie scolaire sur la visite, il nous a paru tout aussi important d'inscrire notre recherche dans l'objectif de valoriser l'éducation non formelle de la visite d'exposition, et de contribuer par la même occasion à rendre compte de la spécificité d'une culture muséale. C'est donc l'expérience de visite dans le cadre des loisirs (en famille) qui a été retenue afin de mieux cerner le phénomène de visite chez les enfants.

Dans le cadre du dispositif médiatique des expositions de sciences, les objets sont mis en contact avec les visiteurs qui établissent des relations

9 « Pensées pendant longtemps en termes d'outils complémentaires - voire alternatifs - de l'école, ces institutions [muséales de sciences et techniques] tendent à être désormais appréhendées en tant qu'outils de culture » (Davallon $1998:$ 399).

10 «Le musée des sciences ne peut que revendiquer la spécificité d'une culture muséale pour échapper à l'emprise de la culture scolaire. [... ] Héritage (fin $20^{\mathrm{e}}$ siècle) d'une muséologie sans professionnels de la médiation ou encore d'une muséologie sans muséologues » (Eidelman,Van Praët 2000 : 7). 
diversifiées selon leurs représentations et leur familiarité (ou non) avec ceuxci. Il s'agit ici de la culture circulant entre des concepteurs d'exposition et des visiteurs, rendue visible à travers leurs pratiques de visite, pour reprendre la définition de la culture selon Y. Jeanneret, comme circulation des objets «entre les mains et les esprits» (Jeanneret 2008 : 17). Étudier la culture comme circulation des objets dans l'espace social de l'exposition revient à s'intéresser à ce que deviennent ces objets ou plus exactement à ce que les visiteurs en font ; comment ils " transforment » leurs représentations de cet objet selon leur subjectivité et mettent en œuvre leur propre compréhension de cet objet. Ces modifications sont précisées par B. Darras (2003 : 21) pour qui le sujet est conduit à approfondir son point de vue, à découvrir d'autres points de vue lorsqu'il profite d'un processus de médiation.

Dans cette perspective, nous avons fait le choix d'observer ce que « fabriquent » les enfants quand ils visitent. Ce qui conduit à inscrire notre recherche dans le cadre de la culture « ordinaire » (selon M. de Certeau 2005), en recueillant la parole auprès des acteurs lorsqu'ils entrent en contact avec l'exposition. Les réactions des visiteurs-enfants et les transformations de leurs représentations du dispositif médiatique de l'exposition apportent un éclairage sur leur expérience de visite, du point de vue phénoménologique, par l'étude des relations qui s'établissent entre les êtres humains et le monde. En contexte muséologique, cette approche est en mesure de contribuer à la compréhension de la construction du « monde utopique » des enfants en portant le regard sur leurs «bricolages » rendus visibles par les relations qu'ils établissent avec le monde «synthétique » de l'exposition recréé par les concepteurs.

Notre travail est en mesure de contribuer d'une part à valoriser l'éducation non formelle face à une « scolarisation » de la visite (Martin, à paraître), d'autre part à montrer comment la visite de l'exposition peut en effet être un « outil culturel » pour de jeunes visiteurs, dans le cadre des loisirs (Martin 2015).

\section{La médiation investie dans le dispositif méthodologique}

Le repérage des qualités du "dispositif " précédemment décrites nous a conduits à mettre en œuvre un dispositif qui requiert ces qualités en réponse à notre questionnement de recherche. Les méthodes traditionnelles utilisées en muséologie n'ont pas donné satisfaction pour rendre compte de l'expérience de visite des enfants, à la fois tout au long du parcours de visite et selon l'ensemble des dimensions qui la traversent. Afin de surmonter ces difficultés, il a été nécessaire de faire preuve d'inventivité en mettant en place un dispositif méthodologique, constituant un « mini-laboratoire » pour observer de manière tangible, les processus d'interprétation dans l'action. La notion de "médiation » a été associée à celle de dispositif. La méthode qui a pour objectif d'attribuer la parole aux enfants s'est concrétisée par l'invention du dispositif de « mise en situation de rôle de guide » précédemment mentionné. Dans cette situation, l'enfant est médiateur en jouant le rôle de " guide » pour 
le chercheur; son interprétation - à l'occasion de cette nouvelle situation de visite - est rendue visible par son récit de médiation, ses gestes, et par ses déplacements dans l'exposition et ses manières de jouer ce rôle, de prendre en considération (ou non) son visiteur.

Comme chacune des méthodes usuelles (observation et entretien) prise isolément ne peut donner entièrement satisfaction pour rendre compte de l'expérience de visite des enfants selon le bilan tiré de notre phase exploratoire, en revanche nous avons décidé de les combiner de manière pertinente pour tirer parti de chacune d'entre elles, dans la perspective de produire de manière rigoureuse une connaissance sur le phénomène de la visite par les enfantsvisiteurs. Nous avions été interpellés par l'affirmation de S. Olivesi portant sur l'intérêt de " mixer et mobiliser des méthodologies diverses selon les nécessités heuristiques de projets de recherche émancipés des routines, des traditions et des contraintes disciplinaires " (Olivesi 2007 : 9). Si bien que le suivi discret d'observation de la visite en famille a été retenu car il permet d'identifier les objets et les manipulations avec lesquels les enfants sont entrés en contact. Lentretien en fin de la visite avec le chercheur permet d'avoir des indications complémentaires sur les visiteurs-enfants (les connaissances des enfants relatives à la thématique et leur provenance, la familiarité avec le lieu, avec cette exposition - la familiarité avec la visite des musées). Par conséquent, ce dispositif méthodologique s'inscrit dans une approche empirique compréhensive (comme définie par A. Mucchielli), ${ }^{11}$ prenant appui sur une démarche ethnographique.

\section{La médiation par le récit comme perspective d'analyse}

À présent nous abordons la médiation d'un point de vue théorique dans le cadre de la démarche d'analyse des résultats obtenus par notre dispositif méthodologique. La mise en situation de rôle de « guide » nous rapproche des travaux de recherche sur la médiation des guides, étudiée par M. Gellereau (2005), dans son ouvrage "Les mises en scène de la visite guidée, communication et médiation ". Dans cet essai sont soulignés les enjeux du récit pour raconter les différentes formes et étapes d'une expérience, pour transmettre des informations. Le récit se fonde sur l'expérience humaine et il devient médiation "parce qu'il lie la visite à l'action humaine dans l'interprétation de la visite " (Gellereau 2005 : 137). De ce fait, le récit permet de réaliser le lien entre le narrateur, le lieu et le destinataire (Gellereau 2005 : 117). Ainsi cet ouvrage développant l'analyse de la visite guidée et l'interprétation des guides apporte un éclairage fondamental pour comprendre la démarche interprétative s'appuyant sur la médiation par le récit et les interactions entre guide et visiteur.

11 Définition du dictionnaire des méthodes qualitatives : l'approche compréhensive est un « positionnement intellectuel (une prise de position épistémologique) [...] qui postule la possibilité qu’a tout homme de pénétrer le vécu et le ressenti d'un autre homme (principe de l'intercompréhension humaine)» (Mucchielli, 2002). 
Bien que les visiteurs-enfants ne puissent être considérés comme de véritables « guides » (dans le sens de guide professionnel), en revanche le rôle de « guide » qui leur est proposé les amène à procéder à une médiation entre l'exposition et leur visiteur, c'est-à-dire à produire des récits par une mise en relation des objets, du lieu, voire de l'institution avec leur visiteur. Si bien que le choix du terrain d'expérimentation - à savoir deux expositions temporaires à thème ayant une trame narrative - est en mesure de constituer un appui pour la construction du récit par les visiteurs-enfants.

Notons que l'importance d'une " trame narrative " (storyline) dans l'organisation conceptuelle de l'exposition a été relevée depuis 1940 par C. E. Cummings, pour faciliter la compréhension de l'exposition par le visiteur $^{12}$. Plus récemment, C. Dufresne-Tassé (2001), considérant qu'il peut exister des types de parcours et de discours plus propices que d'autres à susciter du sens, accorde de l'intérêt à la structure de l'exposition et suggère de s'inspirer de la trame narrative des romans car « la structure des romans pousse progressivement le lecteur à anticiper le déroulement du récit par de petits éléments accrochés successivement les uns aux autres ».

Dans l'exposition "Ombres et lumière ", certains visiteurs-enfants en tant que « guides » commencent par présenter l'exposition en indiquant son propos comme une information primordiale à fournir en début de la visite :

Em : d'abord, c'est sur l'ombre

Et elle précisera au cours de la visite :

$\mathrm{Em}$ : c'est pour montrer qu'on peut faire plusieurs ombres

Em : c'est pour comprendre l'ombre

Des visiteurs-enfants sont en mesure de resituer le propos ou la théorie d'Archibald qui s'y rapporte, aussi bien dans une activité accompagnée d'un cartel que dans une œuvre d'artiste (non associée à un cartel).

\section{La médiation comme concept pour penser les figures des enfants-interprètes}

La médiation par les récits des enfants a permis dans une première phase de mettre en lumière leur interprétation en fonction des relations établies avec chacun des espaces discursifs de l'exposition, et de révéler ainsi les différentes dimensions de leur expérience de visite. Dans une seconde phase, elle a aussi permis de mieux cerner leur démarche interprétative de manière longitudinale, en prenant en considération cette expérience "complète $»^{13}$ vécue par les enfants, sur l'ensemble du parcours de la visite. L'autre piste

12 C'est à partir de là que se sont développés après la Seconde guerre mondiale les expositions à thème et les centres d'interprétation. On retrouve la trame narrative dans les expositions à thème, qui sont souvent des expositions temporaires.

13J. Dewey souligne que « le fait que chaque expérience complète se dirige vers un terme, une conclusion, puisqu'elle cesse seulement quand les énergies qui l'animent ont accompli la tâche qui leur incombe ». (Dewey $2006: 65$ ). 
envisagée pour comprendre comment l'enfant interprète, est la construction de liens pour interpréter. En effet, celle-ci par l'intermédiaire du récit de la " visite guidée " présente un intérêt pour sa "dynamique de se représenter le monde et de construire des liens » (Gellereau 2005 : 117), les liens entre le narrateur, le lieu et le destinataire comme précédemment mentionné. Ceci a conduit, au cours de notre analyse centrée sur la caractérisation de l'interprétation des enfants, à l'élaboration de figures d'enfants-interprètes selon leurs configurations spécifiques. Celles-ci se distinguent à la fois des catégories ou portraits d'enfants, et des figures abstraites comme l'ont proposé D. Jacobi et J. Le Marec (Jacobi, Le Marec 2003 : 47-57) à propos des figures d'interprétation.

Grâce à la situation de "médiateur ", et donc de construction de sens pour d'autres visiteurs (ici le chercheur), il a été possible de structurer les figures des interprètes autour des trois formes de la médiation qui permettent « de penser l'entre-deux de la médiation » et qui « montrent le rôle de la production de sens ", en référence aux travaux sur la médiation de M. Gellereau (2004). Il s'agit de la médiation comme intermédiaire, la médiation comme transition et la médiation comme lien. La médiation comme intermédiaire est définie comme le passage d'un monde à un autre : "Comme "intermédiaire", la médiation est fondée sur l'idée de séparation des mondes et de négociation d'un sens acceptable ou compréhensible par les différents mondes. S'il s'agit d'un dispositif, on pourrait dire qu'il joue un rôle de mise en relation de mondes différents » (Gellereau 2004 : 14). Dans la situation de rôle de « guide », des passages d'un monde à un autre (du réel au fictif, du ludique au sérieux) ont pu être identifiés et étudiés car rendus visibles par l'espace de la performance (en référence à $\mathrm{R}$. Silverstone ${ }^{14}$ ). Ce sont des passages d'un monde à un autre, de leur monde vers le monde de l'exposition, et vers le monde des scientifiques qu'il est possible d'identifier.

La médiation comme transition, qui se manifeste à travers les opérations de mise en relation, entre les objets ou espaces de l'exposition, grâce aux médiations de l'exposition est investie pour analyser la relation des enfants avec les objets, les activités et le lieu. "Comme "transition” à travers les outils et supports de médiation, les formes de médiation, sont ce qui donne accès à une pratique de l'objet qui en permet l'usage ou participe à la formulation de cette pratique ». La médiation de transition sur laquelle peuvent s'appuyer les visiteurs-enfants (comme moyen) regroupe la médiation par le guidage à l'interprétation (cartels et panneaux), la médiation par la mise en scène, la médiation des parents selon les cas (porte-parole du guidage de l'exposition) et leurs commentaires, d'autres formes médiatiques de l'exposition, ainsi que d'autres supports de médiation évoqués par les enfants (livres, films documentaires ou de fiction...).

14 «Dans un espace discursif performatif, les visiteurs sont invités à développer leur propre compréhension et à investir leur propre subjectivité dans le processus d'attribution du sens ». (Silverstone 1998 : 177). 
Un visiteur-enfant en tant que lecteur-débutant s'appuie sur le souvenir de la lecture du cartel par son père afin d'expliquer la consigne d'une manipulation de l'exposition "Ombres et lumière ».

Ju : oui ça veut à peu près dire que voir... que les ombres elles peuvent changer par rapport à...euh...à où elles sont projetées, par exemple l'ombre là ça va pas faire la même chose que l'ombre là..

Ju : c'est papa qui m’a aidé.

La médiation comme lien « permet à chacun de prendre conscience des formes de son rapport au monde et le médiateur ou le dispositif de médiation jouent un rôle intégrateur dans l'institution ou la société au travers de dispositifs qui organisent la relation du sujet au monde »(Gellereau, 2004). Elle est rendue visible à travers les liens qu'établissent les visiteurs-enfants avec l'institution muséale, grâce à leurs façons de la nommer, ou grâce aux critiques formulées sur la mise en scène par exemple.

À propos de la scène de combat entre le T.Rex et l'Ankylosaurus, un enfant considère que le concepteur devrait rendre visibles les morsures sur la queue du dinosaure. Or elle estime que la reconstitution de la réalité comporte des limites car il serait trop difficile de réaliser des trous dans les robots qui seraient constitués d'os.

$\mathrm{Ch}$ : mais non parce qu'ils ont pas pu faire de trous! Ils ont reconstitué avec les os dedans.

On perçoit ici la prise en compte indirecte des concepteurs, par l'emploi du pronom personnel « ils»

$\mathrm{Ch}$ : non, je crois que en fait ils ont mis que de la peinture sur les dents

Ch : oui mais il l'a pas tapé, ils ont juste représenté que les dents rouges ça veut dire (...) qu'il l'a mordu

Les principales composantes retenues pour caractériser leur démarche d'interprétation sont les formes de médiation employées par les enfants (médiation comme transition, comme intermédiaire, comme lien) dans leur rôle de médiateur pour le chercheur ; la prise en compte des espaces discursifs de l'exposition (en observant si l'ensemble des espaces est pris en considération ou si un espace est privilégié) et le type de relation qui en découle. Ce sont ensuite les manières dont les enfants agencent ces différentes composantes et les structurent pour interpréter, qui permettent de comprendre leur démarche d'interprétation et de les conceptualiser. C'est ainsi que les récits de médiation des enfants ont permis l'esquisse de quatre figures d'enfantsinterprètes, pouvant être déclinées selon différentes approches. Il s'agit de la figure de l'enfant-interprète apprenant (constituée à partir de trois 
approches représentant l'envie d'apprendre, à savoir l'observateur détective, l'expérimentateur et le perspicace), la figure du butineur explorateur curieux, la figure du co-constructeur par le partage (partage de connaissances et partage de la sensibilité esthétique), et la figure de l'enfant-interprète inventeur passionné (passionné par la thématique, par la scénographie ou par le jeu). Ces quatre figures et leurs déclinaisons ont pu être identifiées à partir de l'ensemble des dix-neuf cas d'enfants de notre recherche exploratoire, répartis sur deux expositions.

\section{Conclusion}

Pour comprendre l'interprétation des visiteurs-enfants, notre recherche a contribué à "penser la médiation " tout en montrant l'intérêt que revêt sa déclinaison selon des angles d'études diversifiés. La notion de médiation a été associée à celles d'expérience de visite, de construction du sens et d'interprétation, et à celle de dispositif méthodologique heuristique via le rôle de « médiateur ». En portant intérêt à ce que « fabriquent » les enfants dans le cadre de la culture «ordinaire », il a été possible de relever le défi méthodologique par rapport à un public peu étudié dans le cadre des loisirs, grâce à la création d'un dispositif communicationnel s'appuyant sur la médiation des enfants en œuvre dans leur rôle de "médiateur " pour leur visiteur (le chercheur). Par ce dispositif, il a été possible d'accéder à leur interprétation dans l'action. Dans le domaine de la muséologie, la médiation est très riche d'un point de vue heuristique car elle a permis de comprendre la construction de sens des visiteurs-enfants. Elle a été « travaillée » pour permettre l'analyse des récits de médiation recueillis auprès des visiteurs-enfants donnant lieu à l'élaboration des modes d'appropriation de l'exposition et des figures d'enfants-interprètes. Par la même occasion, cela conforte l'idée que cette notion de médiation est bien au cœur des stratégies d'acteurs.

Notre approche de la médiation ainsi présentée nous incline à adhérer aux propos de Jean Caune considérant que «la notion de médiation qui met l'accent sur les processus de contacts, de liens, d'échanges langagiers présents dans le tissu social a une réelle pertinence» (Caune 2010).

\section{BIBLIOGRAPHIE}

BERTEN André (1999), «Dispositif, médiation, créativité : petite généalogie », p. 3347, in «Le dispositif, entre usage et concept ", Hermès, n 25, Éd. CNRS.

CAILLET Elisabeth, LEHALLE Évelyne (1995), À l'approche du musée, la médiation culturelle, PUL, $306 \mathrm{p}$.

CAUNE Jean (2008), Pour une éthique de la médiation: le sens des pratiques culturelles. Pug (Coll. Communication, médias et sociétés), 294 p. 
CAUNE Jean (coord.), « La (les) médiation(s) en SIC », in Les Enjeux de l'Information et de la Communication, Dossier 2010, mis en ligne le 21 décembre 2010, consulté le 8.01.2011. URL : http://w3.ugrenoble3.fr/les_enjeux/pageshtml/art2010-dossier.php

CERTEAU Michel de (2005), L'invention du quotidien. Arts de faire 1, Paris: Folio essais, $416 \mathrm{p}$.

DARRAS Bernard (2003), «Entretien avec Bernard Darras par Marie Thonon », in MEI, $\mathrm{n}^{\circ} 19$, sous direction Marie Thonon, Médiations et médiateurs, p. 16-29.

DAVALLON Jean (1992), "Le musée est-il vraiment un média ? ", in Publics E̋ Musées. $\mathrm{n}^{\circ}$ 2, p. 99-124.

DAVALLON Jean (2003), Pourquoi considérer l'exposition comme un média ? Médiamorphoses, n' 9 , p. 25-28

DAVALLON Jean (2005), L'exposition à l'œuvre : stratégies de communication et médiation symbolique. L'Harmattan, $378 \mathrm{p}$.

DELARGE Alexandre, (2001), « Pratiques interprétatives en muséologie », in Étude de communication : langages, information, médiations, $\mathrm{n}^{\circ} 24$, L'interprétation : entre élucidation et création, p. 57-69.

DEWEY John, (2006), Les cuvres philosophiques de John Dewey. L'art comme expérience III. Université de Pau. Farrago, Traduites de l'anglais (USA) par COMETTI Jean-Pierre, DOMINO Christophe, GASPARI Fabienne.

DUFRÊNE Bernadette, GELLEREAU Michèle (2004), « La médiation culturelle : enjeux professionnels et politiques ", in Les Sciences de l'Information et de la Communication. Entre science et professionnalisation, n 38, Hermès, CNRS éditions, p. 199-206.

DUFRESNE-TASSÉ, Forum Université de Montréal. L'exposition comme un roman. Volume 35 numéro 26 - 2 avril 2001. Disponible sur : http://www.forum.umontreal.ca/ numeros/2000_2001/forum_01_04_02/article10.html (consulté le 24.04.2011)

GELLEREAU Michèle (2004), Construire un monde culturel commun. Pratiques langagières et formes de médiation, vol. 1, Parcours de recherche et synthèse des travaux. Habilitation à diriger des recherches en Sciences de l'Information et de la Communication. ss la dir. de DELFORCE, Bernard. Université Lille 3.

GELLEREAU Michèle (2005), La mise en scène de la visite guidée : communication et médiation. Paris : L'Harmattan, 279 p.

GELLEREAU Michèle (2007), "Pratiques culturelles et médiation ». In Sciences de l'information et de la communication : objets, savoirs discipline. ss la dir. OLIVESI, Stéphane, Pug, p. 31-42.

HENNION Antoine, MAISONNEUVE Sophie, GOMART Emilie (2000), Figures de l'amateur, formes, objets, pratiques de l'amour de la musique aujourd'bui. La Documentation Française. 281 p.

JACOBI Daniel, SCHIELE Bernard (1990), « La vulgarisation scientifique et l'éducation non formelle ", in Revue française de pédagogie, vol. 91, n 1. p. 81-111.

JACOBI Daniel, LE MAREC Joëlle (2003), « Sur quelques figures de l'interprétation dans la culture ", in L'interprétation : objets et méthodes de recherche. Ss la dir. de LA BROISE, Patrice. Actes du Colloque du 11 mai 2000 aux Archives du Monde du Travail, 
Roubaix. Université Lille 3 : Édition du Conseil Scientifique de l'Université Charles de Gaulle, p. 47-57.

JEANNERET Yves (2008), Penser la trivialité, vol. 1, La vie triviale des êtres culturels. Éditions Hermès-Sciences-Lavoisier.

LAMIZET Bernard, SILEM Ahmed (1997), «Médiation», in Dictionnaire encyclopédique des sciences de l'information et de la communication, Ellipses, p. 436-437.

MARTIN Thérèse (2011), L'expérience de visite des enfants en musée de sciences dans le cadre des loisirs : les logiques d'interprétation et enjeux d'un dispositif communicationnel. Thèse en Sciences de l'Information et de la Communication. Sous la direction de Michèle Gellereau Université Lille 3. Disponible sur http://tel.archivesouvertes.fr/tel-00783563

MARTIN Thérèse (2012), Les logiques d'interprétation des enfants selon leur expérience de visite dans les musées de sciences et dans le cadre des loisirs. In Communication, volume 30/2, Université Laval, Québec. Disponible sur : http:// communication.revues.org/index3598.html

MARTIN Thérèse (2015), Motivations pour la mise en lumière la culture muséale des enfants : un enjeu de valorisation de l'éducation non formelle. Éducation et francophonie. Éducation muséale. Québec. 2015. Disponible sur http://www.acelf.ca/c/revue/ toiledefond.php?id=34\#.VT1RMGaI0vo

MARTIN Thérèse, « Des figures d'enfants-interprètes dans le cadre des loisirs, sources d'inspiration pour la visite scolaire et les modes de transmission », In Actes des journées d'études De l'école au musée : quelles conceptions des savoirs, quels modes de transmission? De l'espace scolaire à l'espace muséal, la transmission par l'expérience vécue. École du Louvre, 31 janvier 2014, Paris. (Actes en attente de publication)

OLIVESI Stéphane, (2007), "Introduction », in OLIVESI S. dir., Introduction à la recherche en SIC, PUG, p. 5-10.

SILVERSTONE Robert (1998), «Les espaces de la performance : musées, science et rhétorique de l'objet » in Hermès, $n^{\circ}$ 22, La mimésis, p. 175-188.

TILDEN Freeman (1957), Interpreting our heritage, Chapell Hill, University of North Carolina Press.

VÉRON Eliséo (1983), L'exposition comme média in Histoire d'exposition Peuple et culture, centre de création industrielle, Centre George Pompidou, p. 41-43.

VÉRON Éliséo, LEVASSEUR Martine (1991), Ethnographie de l'exposition, Études et recherche, Centre Georges Pompidou, Bibliothèque publique d'information, 206 p.

Résumé : Cet article vise à montrer comment notre recherche qui se situe dans le cadre de la médiation culturelle, plus précisément dans le contexte muséologique de sciences a eu recours à la médiation afin de comprendre le phénomène de visite d'exposition chez les enfants dans le cadre de loisirs, et ceci à deux niveaux. Du point de vue méthodologique, la médiation des enfants en tant que " guides » est l'élément central du dispositif communicationnel inventé pour mettre en évidence l'interprétation des enfants in situ. 
Comme concept, la médiation rend possible l'analyse de l'interprétation des enfants via leurs récits et leurs gestes, et permet - à partir des relations établies avec le monde de l'exposition - l'élaboration de quatre figures d'enfants-interprètes et de leurs déclinaisons, qui révèlent les modes d'appropriation de l'exposition par les visiteurs-enfants.

Mots-clés : médiation, interprétation, public-enfants, expérience de visite, dispositif méthodologique, culture muséale, exposition de sciences.

\begin{abstract}
The aim of this article is to show how our doctoral research, anchored in the framerwork of cultural mediation, particularly within the context of science museum, has to resort to mediation in two respects. From a methodological point of view, the mediation of the children as "guides» is the pivotal element in the communicational device created to characterize the interpretation of the children in situ. From a conceptual point of view, medation contributes to analysing the children's speech narratives. The analysis has thus enabled us to identify four types of children-interpreters and to characterize how each of them illustrates different exhibition appropriation methods.
\end{abstract}

Keywords : mediation, interpretation, the public and children, visit experience, methodological device, cultural museum, science exhibits. 